\title{
Mobile Monitoring System for Gas Sampling Based on Zigbee, 4G and Web GIS
}

\author{
Zhanying Zhang ${ }^{1, \text { a) }}$, Congcong Du ${ }^{1}$, Yang Liu ${ }^{1}$ and Lixia Han ${ }^{2}$ \\ ${ }^{1}$ College of Computer and Information Engineering, Tianjin Normal University, Tianjin 300387, China. \\ ${ }^{2}$ The People's Procuratorate of Tianjin Xiqing District, Tianjin 300380, China. \\ a) Corresponding author: zhanying999666@163.com
}

\begin{abstract}
For the realization of the real-time collection, upload, visualization and intelligent management of air quality data, mobile management system of gas sampling was designed based on the technologies including Web GIS, ZigBee wireless sensor network, the 4G (4th generation) communication system and so on. The ZigBee module is mainly used for wireless communication between PDA and mobile sensor nodes. The 4G module is used to upload data uploaded by ZigBee terminal nodes to the remote server through TCP/IP protocol, to realize remote monitoring of spatio-temporal variation of gas information. The real-time tracking of gas sampling can be realized through Digital Map, with the combination of GPS information and Web GIS technique. The spatial distribution figure of gas sampling can be generated automatically by calling data stored in database. It can guide the the follow-up of manual decision effectively.
\end{abstract}

Key words: Web GIS; ZigBee wireless; database; 4G (4th generation)

\section{INTRODUCTION}

Due to traditional hand-held environmental monitoring equipments have space limitation, unable to monitor the environment of a narrow space and a dangerous place. This study is mainly for experimental car for gas sampling. In this study, an intelligent vehicle equipped with monitoring equipment of gas sampling was designed. Mobile acquisition nodes networking through the ZigBee wireless sensor network.

ZigBee technology is a wireless network communication technology with low power, low complexity, low transmitted rate, low cost, reliable communication and short distance communication, working on the $2.4 \mathrm{GHz}$ ISM band. ZigBee wireless network is based on the IEEE802.15.4 standard, defines the MAC layer and PHY layer of the protocol. ZigBee protocol has been perfected and expanded on the basis of IEEE802.15.4, and defines the high level of the system, including network layer, application layer and security service provider layer[1].Its architecture is shown in Fig1(a).ZigBee network is an achieve simple Ad Hoc Network, and no manual configuration is required for new sensor nodes to join the network. It is widely used in wireless environment monitoring system. Star topology, tree topology and mesh topology can be built by using ZigBee technology with powerful networking capability. Its network topology is shown in Fig1 (b). 


\begin{tabular}{|c|c|c|}
\hline \multicolumn{2}{|c|}{ Application Layer } & Customer \\
\hline Applicat io & nterface & \multirow{3}{*}{$\downarrow$} \\
\hline \multicolumn{2}{|c|}{ Security Layer } & \\
\hline \multicolumn{2}{|c|}{ Network Layer } & \\
\hline \multicolumn{2}{|c|}{ IEEE802. 15. 4MAC } & \multirow{3}{*}{ IEEE 802.15.4 } \\
\hline IEEE802. 15. 4 & IEEE 802.15 .4 & \\
\hline $868 / 915 \mathrm{MHz}$ PHY & 2. $4 \mathrm{GHz} \mathrm{PHY}$ & \\
\hline
\end{tabular}

(a)

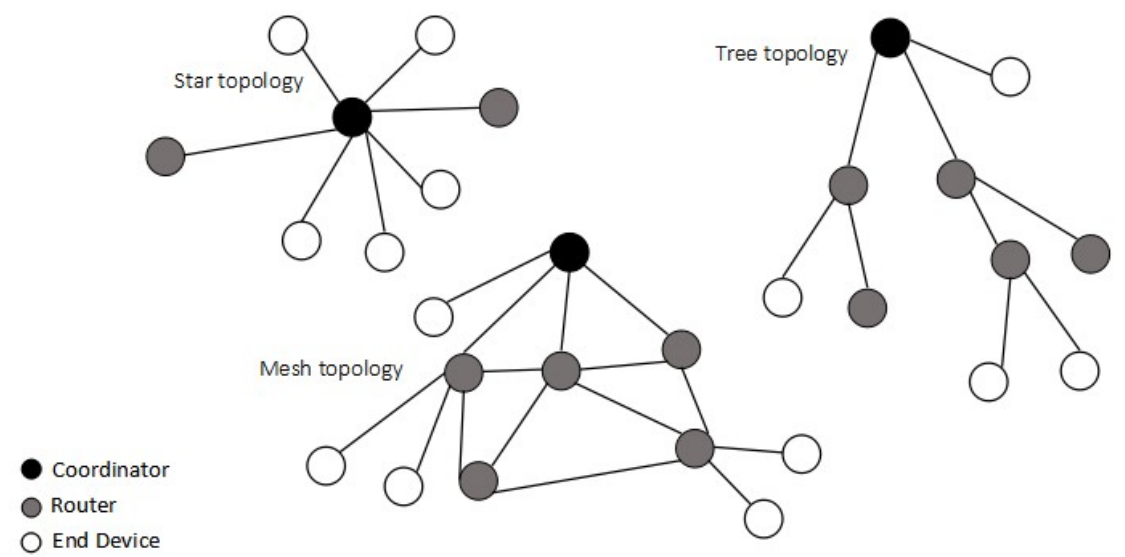

(b)

FIGURE 1. Architecture and network topology of the ZigBee protocol

The purpose of this study is to design a safe, efficient and personalized mobile management system of gas sampling by using the technologies including ZigBee wireless sensor network, the 4G communication system, Web GIS, spectral analysis and so on. A lot of scholars at home and abroad have designed some environmental monitoring systems by using WSN. The representative results are as follows: Wang Dehui and others proposed a remote monitoring and management system based on wireless sensor network and 3G/4G[2].Chen Dingyi and others designed a monitoring platform of soil sampling based on barcode technology and Web GIS technology[3].Sun Jimin and others studied the method of WSN to connect Internet[4].Wang Xingming and others proposed a soil sampling remote monitoring management system based on wireless technology and $3 \mathrm{~S}$ technology[5]. The above research results lack of the most advanced implantion of new technology, such as cloud computing and Android, lack of advanced design idea, and scalability needs to be enhanced. In view of these weaknesses, this paper has carried out research and improvement, a gas sampling remote monitoring and management system based on ZigBee wireless technology was designed.

\section{SYSTEM FRAME DESIGN}

This study is mainly for experimental car for gas sampling. The system consists of ZigBee acquisition node, gateway of the coordinator, mobile terminal PDA and remote management software. The whole framework for the mobile monitoring system is shown in Fig 2.The ZigBee network is responsible for the collection and upload of data. 


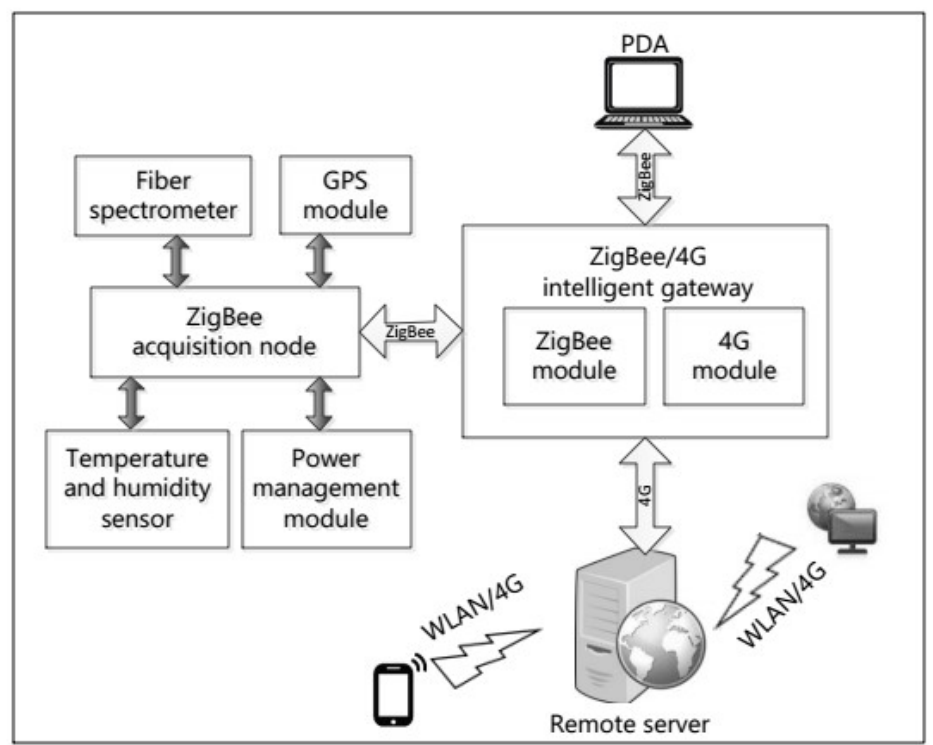

FIGURE 2. The system block diagram

ZigBee acquisition node is mainly responsible for sensor data acquisition, consists of ZigBee terminal node, temperature and humidity sensor, PS module and fiber spectrometer. Acquisition node sends the collected GPS information, temperature and humidity data, gas wavelength concentration data to mobile terminal PDA through ZigBee network. The data of acquisition node would be transmitted to management software of remote server through the $4 \mathrm{G}$ network after be transformed from the ZigBee wireless network to $4 \mathrm{G}$ network. Temperature and humidity sensor is used for measuring of the air temperature and humidity information in the sampling area. The GPS module is used to obtain latitude and longitude information so that the sampling point real-time tracing system can be built and the spatial map of the gas elements ,the map of area monitoring and so on can be drawn. Fiber spectrometer is mainly responsible for spectra collection, processes the collected spectral signal and finally obtains the data result. The data structure of the spectrometer is wavelength-concentration. Intelligent gateway includes the ZigBee module and the $4 \mathrm{G}$ module, implements the conversion between the ZigBee protocol and the $4 \mathrm{G}$ communication protocol. ZigBee/4G heterogeneous network rely on intelligent gateway for protocol conversion, so that the on-site ZigBee data packets can be re-encapsulated then uploaded to the remote server through TCP/IP protocol.

\section{SYSTEM HARDWARE DESIGN}

\section{Design of ZigBee Wireless Sensor Network}

ZigBee terminal node is also a sensor information acquisition terminal, the temperature and humidity sensor and the GPS module are connected to the ZigBee module through the I2C bus and the RS232 serial port respectively, and fiber spectrometer is connected to the ZigBee module via USB serial port. The acquisition node packs sensor information into ZigBee packets and then sends it to the gateway via ZigBee wireless network. The intelligent gateway then uploads the data to the remote server through the $4 \mathrm{G}$ network. The hardware structure of the mobile acquisition node mainly includes the sensor acquisition module, the wireless communication module, the video recording module, the spectrometer module, the GPS module and the power management module.

(1) The Sensor Acquisition Module

In the equipment, the DB4O5A temperature and humidity sensor (Sensation AG Switzerland) is selected for the acquisition node. Range from $-20^{\circ} \mathrm{C}$ to $80^{\circ} \mathrm{C}$ and precision $\pm 0.3\left(25^{\circ} \mathrm{C}\right)$ for temperature. Range from 0 to $100 \% \mathrm{RH}$ and precision $\pm 3 \% \mathrm{RH}$ for humidity.

(2) The Wireless Communication Module 
The wireless communication module is responsible for the ZigBee network construction and data transceiver. The JN5139 module is used as wireless communication module between the PDA and the mobile sensor acquisition nodes. It integrates a 32-bit RISC processor and a fully-compatible 2.4GHz IEEE802.15.4 transceiver, 192kB ROM,96kB RAM, various digital and analog peripherals.32-bit data operation is very effective for protocol stacks and high-end applications. It allows algorithms to be executed with fewer instructions compared to the short wordlength processor that means fewer clock cycles are required to execute the same algorithm. The JN5139 module integrates the RF component, saves development time and cost.

(3) The Video Recording Module

(4) The Spectrometer Module

The USB2000+ module (Ocean Optics, USB series) is selected as the spectrometer module. The USB2000+ integrates a 2-MHz A/D Converter programmable electronics, a 2048-Element CCD-Array Detector and a USB2.0 Communication. The module covers a range of 200-1100nm, and can be connected to light source, colorimetric dish and other accessories.

(5) The GPS Module

The GPS module uses the OEM615 module (Novatel, Canada), that can realize the real-time positioning of the sampling source.

(6) The Power Management Module

\section{Design of Intelligent Gateway}

In the design of the gateway, considering from the view of technology how to implement the WSN remote effective communication through the bidirectional interaction and the seamless connection between the ZigBee node and the $4 \mathrm{G}$ communication system[6]. The gateway is responsible for the conversion between the ZigBee wireless communication network and the $4 \mathrm{G}$ mobile communication and it consists of the ZigBee module(ZigBee coordinator node) and mobile communication module. The details are as follows: 1)The ZigBee coordinator is responsible for the networking of the ZigBee network. It receives data from the acquisition node, and sends instructions from the PDA or remote management software to the terminal node, and it connects the mobile communication module through the serial port, complete the Mixed Networking of ZigBee/4G.On the hardware, the ZigBee coordinator is basically the same as the acquisition node, both achieve their respective functions through software programming, flowcharts are shown in Fig3(a)(b).(2)Mobile communication module realizes remote mobile communication through Socket network programming.
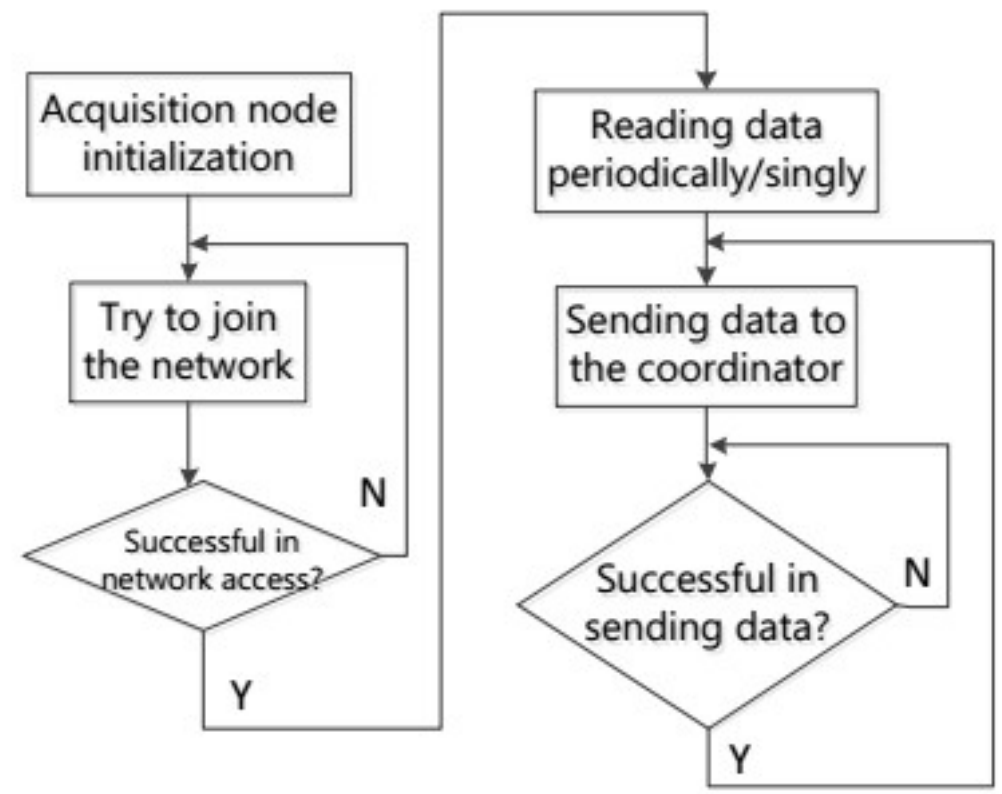

(a) 


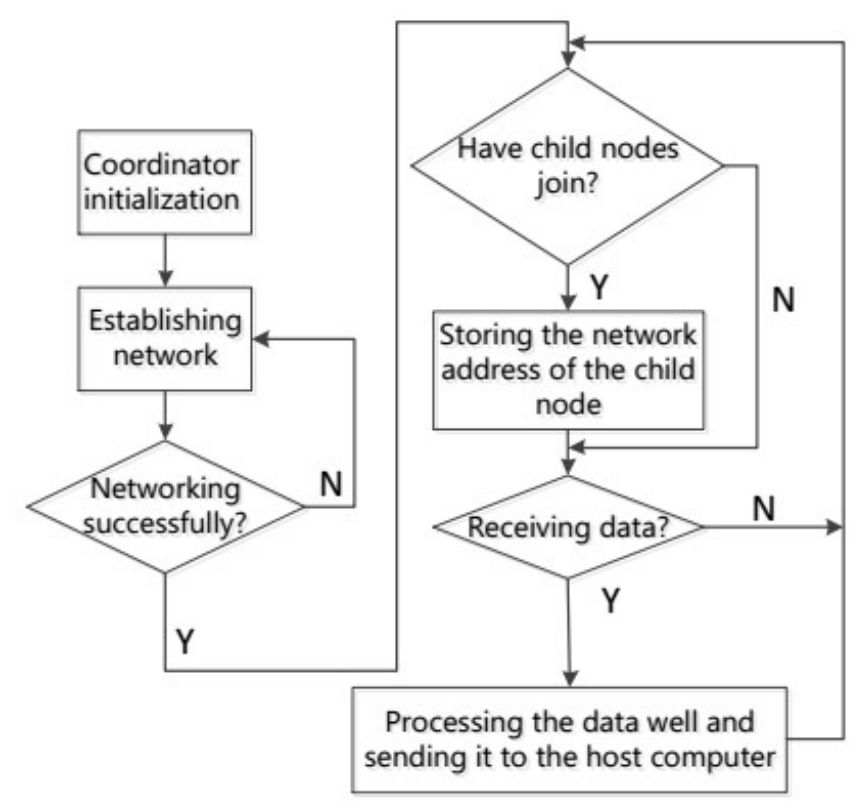

(b)

FIGURE 3. The flowchart of terminal nodes and coordinator

\section{Design of Field Mobile Terminal PDA}

The main functions of the PDA include:(1)The bidirectional communication with mobile sensor nodes. (2) It receives and saves the GPS information, temperature and humidity information and spectral analysis data uploaded by the acquisition node, so as to realize the real-time display and storage data of the PDA. The PDA is mainly composed of the main functional module and the ZigBee module. The ZigBee module connects the main functional module through the serial port. The main functional module of the PDA adopts the S3C2442A of the ARM 920T series. The ZigBee module is a streamlined ZigBee coordinator, that is mainly responsible for receiving data from the acquisition nodes.It communicates with the main functional module of the PDA through the serial port and the main chip uses the JN5139-M03 uFI antenna module.The ZigBee module occupies the COM4.

\section{SYSTEM SOFTWARE DESIGN}

\section{Software Design of Mobile Acquisition Node}

The software of mobile terminal nodes mainly includes three parts: initialization process, instruction configuration of sensor sampling, sampling and sending of sensors[7].The working condition of each module of the mobile terminal node during the process of data acquisition and wireless transmission is shown in Fig4. The concrete steps are as follows: (1)The first step is to initialize, that includes the initialization of peripherals and the initialization of the ZigBee protocol stack, the next step is to do the channel query, automatically find and join the network, then the CPU enters the dormant period with waiting for the event-triggered.(2)When the network coordinator sends commands of the PDA to the terminal nodes through the ZigBee network. The CPU is triggered. Temperature and humidity sensor, the GPS module and spectrometer module collect data information and send it to the coordinator through the ZigBee network.(3)The coordinator forwards data to the PDA.The ZigBee packet is repackaged by the intelligent gateway and then be uploaded to the remote server via the $4 \mathrm{G}$ network. 


\section{Software Design of Remote Server Management Platform}

The remote management software of this study is an intelligent management platform for gas monitoring based on the Web GIS. It integrates the Java2EE language technology, MySQL database storage and the Web GIS technology, and the function structure is shown in Fig5.The development environment of this study is Intellij and the development language is Spring Boot [8].

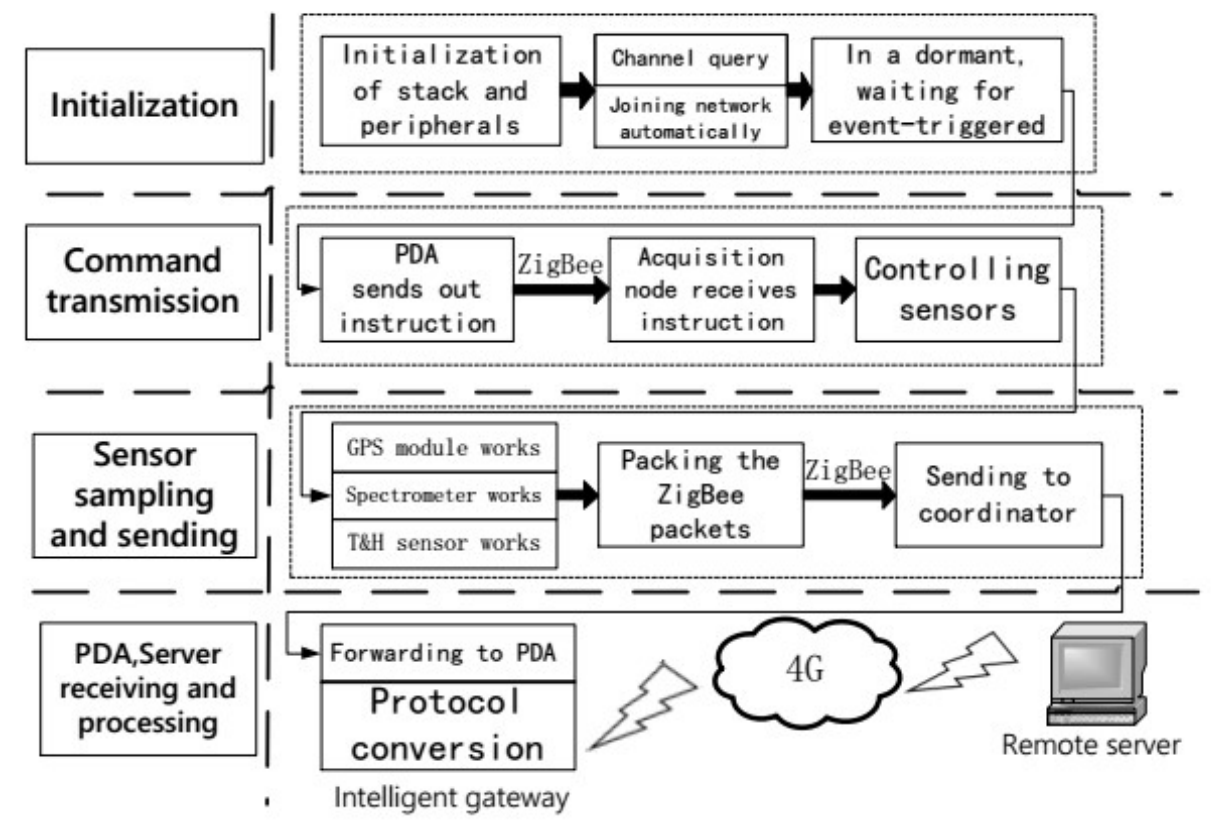

FIGURE 4. Flow chart of data acquisition and wireless transmission

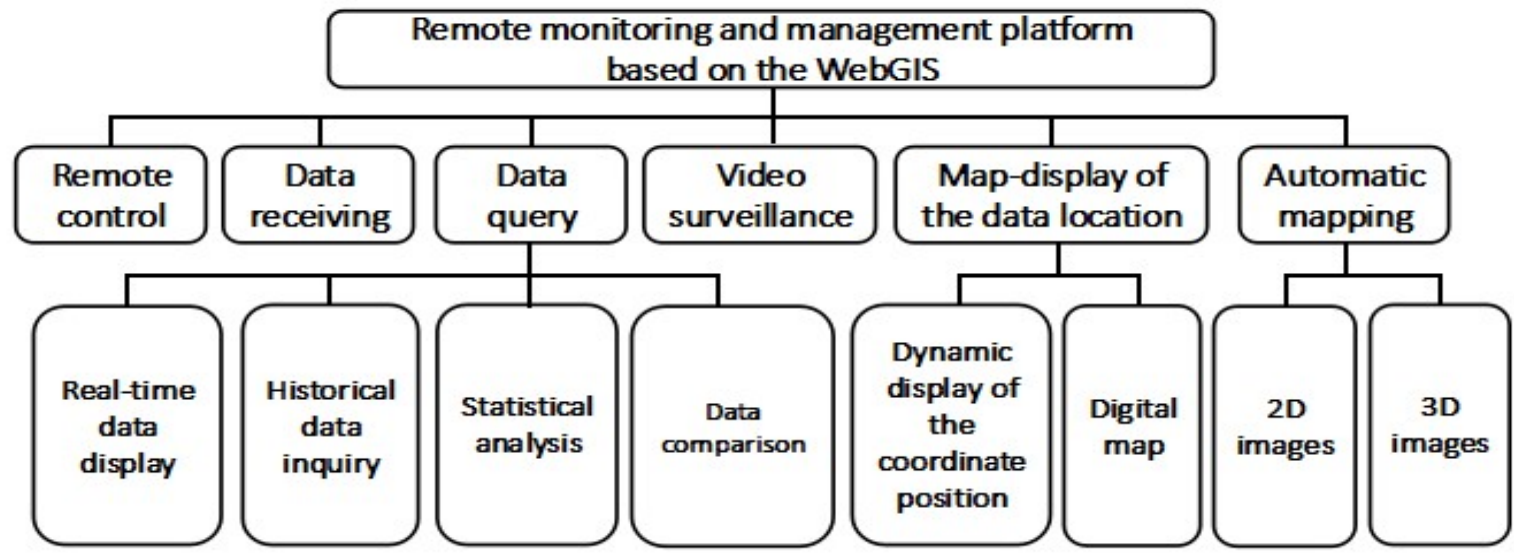

FIGURE 5. Function module design of remote management software

The research platform carries out data fusion between the real-time monitoring data of sensors and the WebGIS spatial data, thus the accurate location of sampling source and dynamic monitoring of sampling area are realized. A spatial database is established, that is used to record spatial data, such as the topological relations of map point-linesurface and other space elements, the position coordinates of monitoring points, as well as the raster data of remote sensing maps of monitoring area. The WebGIS server can perform spatial retrieval and spatial analysis with the comprehensive treatment of spatial data. That is, on the geographic map based on remote sensing imaging, the 
vector layers are covered, such as the position information of sampling source, temperature and humidity information, gas element information and so on. The WebGIS server relies on vector layers to accomplish spatial analysis, generate the Digital Map dynamically, then real-time map display and timing stored in the spatial database. The application of the WebGIS in this system is mainly the map display of data location, that includes map positioning of sampling sources, as well as real-time and on-line display of monitoring data .Researchers can view the location information of the monitoring sources, data distribution, equipment operational condition and so on real-timely through the map browsing operation. Users can choose to display all the data, or choose to display some region or some type of monitoring data.

\section{SYSTEM PERFORMANCE ANALYSIS AND TEST}

\section{System Stability Test}

Eliminating the problem of hardware interference before testing, such as bad contact, short circuit, hardware loosening or power supply instability, etc. Then do electrifying and debugging. During the test, the Mesh topology is selected as network topology. The sensor network based on the ZigBee protocol is relatively stable, but the system is also disturbed by obstacles and severe weather, such as lightning, rain and snow, sandstorm, tornado, etc, so an open area is chosen for testing. The temperature of test site is $28^{\circ} \mathrm{C}$, the humidity is about $41 \%$. The distance between mobile acquisition node and the PDA is $50 \mathrm{~m}$, and the distance between the test site and the remote server is about $31.8 \mathrm{~km}$. With continuous observation of test results, the number of lost packets and the packet loss rate are shown in Table1.The temperature and humidity chart in the remote management software is shown in Fig6.

The experimental results show that the coordinator node can receive the data sent by the acquisition node in real time, and the intelligent gateway realizes the functions of ZigBee/4G protocol conversion and uploading data. By comparing with the actual temperature and humidity, it proves that the collected data is basically accurate, the packet loss rate is only $0.3 \%$, the system can work steady and reliably. As the experiment went further, it was found that the maximum communication distance of data transmission is about $200 \mathrm{~m}$, it meets the system requirements. With the increase of communication distance, the packet loss rate is increasing constantly, so the packet loss rate is proportional to the communication distance.

TABLE 1. Packet loss number and rate of the system

\begin{tabular}{ccccc}
\hline Sending terminal & $\begin{array}{c}\text { Receiving } \\
\text { terminal }\end{array}$ & $\begin{array}{c}\text { Number of sending } \\
\text { packets/PCS }\end{array}$ & $\begin{array}{c}\text { Number of receiving } \\
\text { packets/PCS }\end{array}$ & $\begin{array}{c}\text { Number of lost } \\
\text { packets/PCS }\end{array}$ \\
\hline $\begin{array}{c}\text { Mobile } \\
\text { acquisition node } \\
\text { Mobile }\end{array}$ & PDA & 1000 & 997 & 3 \\
acquisition node & Remote server & 1000 & 997 & 3 \\
\hline
\end{tabular}
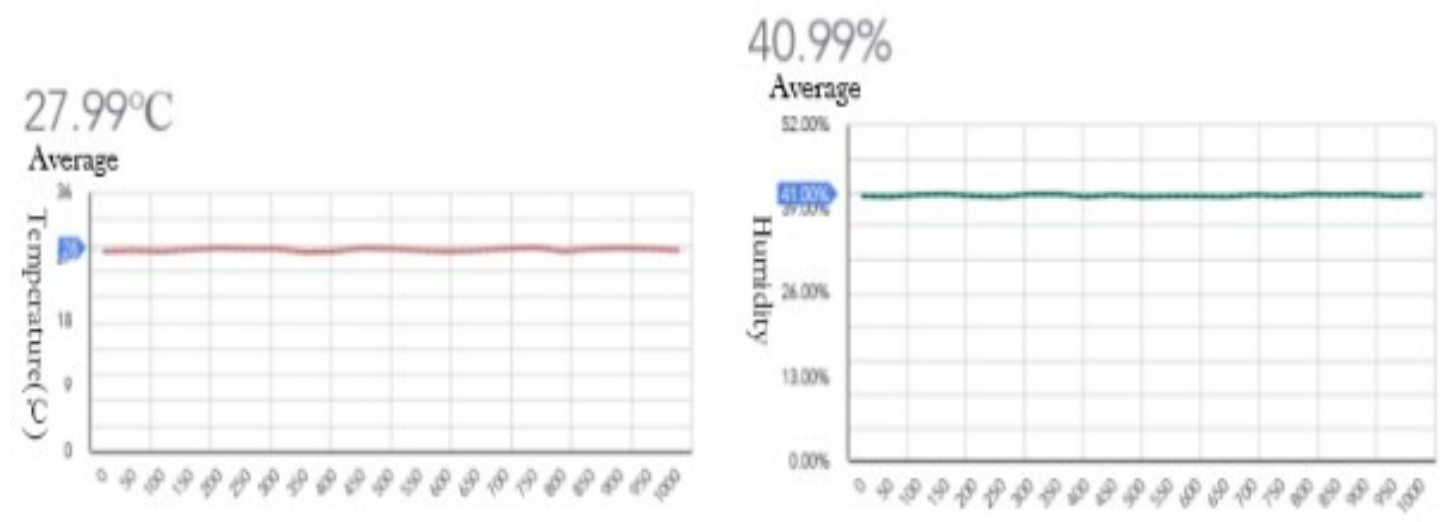

FIGURE 6. Temperature and humidity images 


\section{System Application Experiment}

The application test of the system was held in an open area of Jingwu Town, Xiqing District, Tianjin on August 17, 2017.Place the mobile acquisition node near the selected sampling point, then use the PDA to command car to collect and upload the corresponding GPS information, temperature and humidity information and spectral analysis data, and the data is uploaded to the remote server by the intelligent gateway. Then use the PDA to direct the car to other sampling points. The data uploaded to the server over the 4G network is exactly the same as the data stored in the PDA. The dynamic display interface of the spatial distribution for sampling points at a certain time is shown in Fig 7. The background is the geographic map of the experimental region and the positioning icons indicate the location of the sampling points. Click any positioning icon to display the acquisition time, GPS information, temperature and humidity data and reference position of this sampling point. The server management software combines with the data uploaded by the acquisition node in the database and generates images automatically. The spatial distribution map of air humidity generated by the comprehensive treatment of relative humidity and GPS information is shown in Fig 8

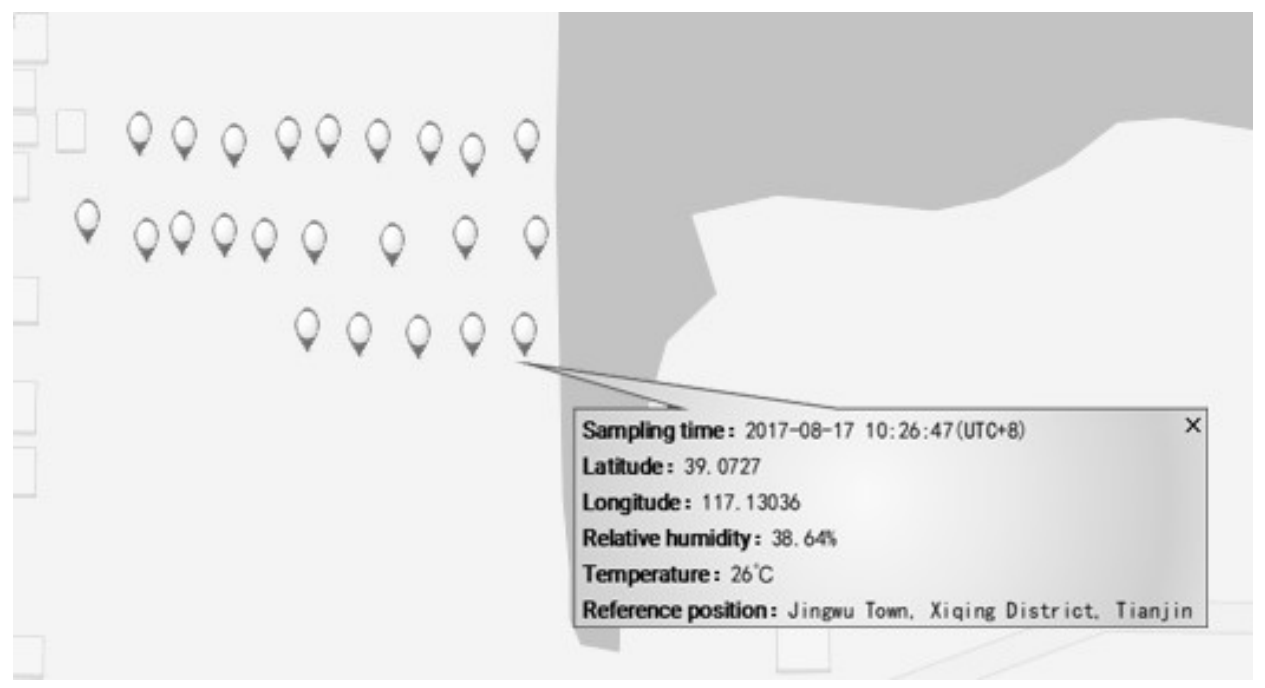

FIGURE 7. Dynamic display interface of spatial distribution for sampling points

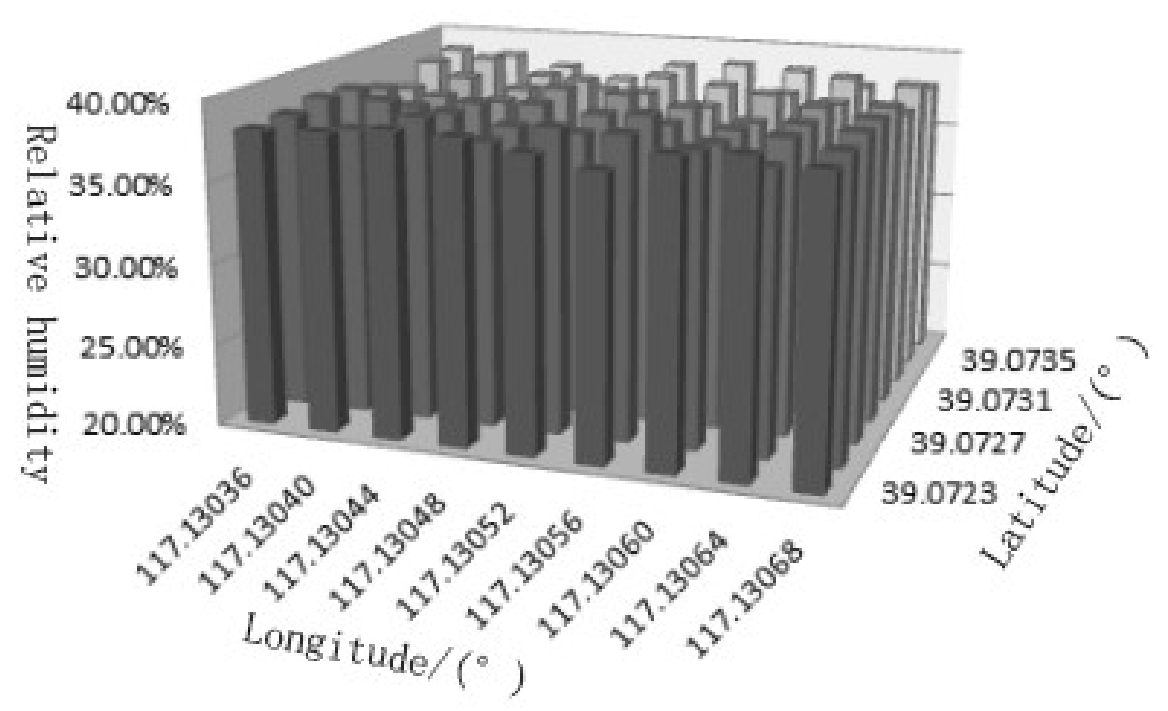

FIGURE 8. Spatial distribution map of air humidity 


\section{CONCLUSION}

This paper designs and develops the hardware and software of the mobile monitoring system for gas sampling based on the ZigBee, 4G and WebGIS. The system is designed and developed based on the ZigBee protocol, and it realizes the wireless monitoring of temperature, humidity and gas elements in the environment. The sampling data can be stored synchronously in the PDA and remote server, users can browse real-timely through web pages or Android devices, and it has good visibility and portability. The integration of mobile communication, digital map and other technologies makes the system more practical and advanced. The system is designed based on the ZigBee network, that is simple and easy to implement .In the process of data sampling and data transmission, the system runs steadily and the data is unmistakable, it can be widely used in air quality monitoring, coal mine safety monitoring, probe of dangerous environment and other fields.

\section{ACKNOWLEDGEMENTS}

The reported study was funded by the Doctorate Fund (research project 52XB1203) and the Research on the Countermeasures to Promote Scientific and Technological Innovation in Tianjin's Intelligent Transportation Field (research project 17ZLZXZF00530).

\section{REFERENCES}

1. Zhang Wenshu, Duan Dongliang, Yang Liuqing. Relay selection from a battery energy efficiency perspective [J]. IEEE Transactions on Communications, 2011, 59(6):1525-1529.

2. D.Duan, F.Qu, L.Yang, A.Swami,and J. C. Principe. Modulation Selection from a Battery Power Efficiency Perspective [J]. IEEE Transactions on Communications, vol. 58, no.7, pp. 1907-1911, July 2010.

3. Riliskis, L. Osipov,E.Symphony. A Framework for Accurate and Holistic WSN Simulation [J]. Sensors, 2015, 15:4677-4699.

4. Arch Rock Corp.San Francisco. IPv6 in Low-Power Wireless Networks [J]. Proceedings of the IEEE, 2010; 98(11):1865-187.

5. Atzori L,Iera A, Morabito G. The internet of things: A survey [J]. Computer Networks, 2010, 54(15): 27872805 .

6. Jula A, Sundararajan E, Othman Z.Cloud computing service composition: A systematic literature review [J]. Expert Systems with Applications, 2014, 41(8):3809-3824.

7. Wei Qingfeng, Luo Changshou, Sun Sufen, et al. Present status and thoughts about agricultural information service based on cloud computing in China [J]. Journal of Agricultural Science and Technology, 2013,15(4): 151-155. (in Chinese with English abstract)

8. Xu Shiwei, Wang Dongjie, Li Zhemin. Application research on big data promote agricultural modernization [J]. Scientia Agricultura Sinica, 2015, 48(17):3429-3438. (in Chinese with English abstract) 